\title{
Adult classical glioblastoma with a BRAF V600E mutation
}

\author{
Yoshinobu Takahashi ${ }^{1,3^{*}}$, Toshiaki Akahane ${ }^{2,3}$, Takahiro Sawada ${ }^{3}$, Hidetoshi Ikeda ${ }^{1}$, Akira Tempaku ${ }^{1,3}$, \\ Shigeru Yamauchi ${ }^{1}$, Hiroshi Nishihara ${ }^{2,3,4}$, Shinya Tanaka ${ }^{4}$, Kazumi Nitta', Wataru Ide ${ }^{1}$, Ikuo Hashimoto ${ }^{1}$ \\ and Hajime Kamada'
}

\begin{abstract}
The B-Raf proto-oncogene serine/threonine kinase (B-Raf) is a member of the Raf kinase family. The BRAF V600E mutation occurs frequently in certain brain tumors such as pleomorphic xanthoastrocytoma, ganglioglioma, and pilocytic astrocytoma, and less frequently in epithelioid and giant cell glioblastoma. BRAF V600E mutation in these cases has been canonically detected using Sanger sequencing or immunohistochemistry but not with next-generation sequencing (NGS). Moreover, to our knowledge, there is no detailed report of the BRAF V600E mutation in an adult glioblastoma with classical histologic features (c-GBM). Therefore, we performed NGS analysis to determine the mutational status of BRAF of 13 glioblastomas (GBMs) (11 primary and 2 secondary cases) and detected one tumor harboring the BRAF V600E mutation. We report here the detection of the BRAF V600E mutation in a patient with c-GBM and describe the patient's clinical course as well as the results of histopathological analysis.
\end{abstract}

Keywords: BRAF V600E, Adult, Classical glioblastoma

\section{Background}

The B-Raf proto-oncogene serine/threonine kinase (BRaf) is a strong activator of the extracellular signalregulated kinase/mitogen-activated protein kinase 1 and 2 (Erk 1/2) signal transduction cascade that mediates cell proliferation [1]. Most BRAF mutations occur at codon 600 , which resides within the activation loop of the kinase domain, and $80 \%$ to $90 \%$ of these mutations generate a protein with a glutamic acid (E) residue substituted for the normal valine $(\mathrm{V})$ residue (BRAF V600E). Such mutant proteins exhibit increased kinase activity and transform cultured cells. The BRAF V600E mutation occurs frequently in certain brain tumors such as pleomorphic xanthoastrocytoma (PXA) (60\%), PXA with anaplastic features (60\%), ganglioglioma (20\% to $60 \%)$, extracerebellar pilocytic astrocytoma (20\%) [2-5], epithelioid glioblastoma (54\%) [6], and giant cell glioblastoma (7\%) [5]. However, the few studies of adult

\footnotetext{
* Correspondence: yosinobu@hokuto7.or.jp

'Department of Neurosurgery, Hokuto Hospital, 7-5, Inada, Obihiro, Hokkaido 080-0039, Japan

${ }^{3}$ Department of Biology and Genetics, Laboratory of Cancer Medical Science, Hokuto Hospital, 7-5, Inada, Obihiro, Hokkaido 080-0039, Japan

Full list of author information is available at the end of the article
}

classical glioblastoma (c-GBM) with the BRAF V600E mutation lack detailed characterization of the tumors. Here, we present the first report, to our knowledge, that combines histopathological, immunohistochemical, and next-generation sequencing (NGS) analyses of c-GBM with the BRAF V600E mutation.

\section{Case presentation}

A 49-year-old man was admitted to the hospital complaining of headache, vomiting, and mild left hemiparesis. Magnetic resonance imaging (MRI) showed a huge multicystic mass in the right occipitoparietal area with marked surrounding edema and a shift of the midline structures to the left side (Figure 1A). The cyst wall and adjacent cortical mass were enhanced with contrast medium (Figure 1B). 18F-Fluorodeoxyglucose (FDG) and methionine (MET) positron emission tomography (PET) revealed high accumulation in the right occipitoparietal area (Figure 1C, D).

Near-total resection of the tumor was performed. After glioblastoma (GBM) was pathologically diagnosed, the patient had local radiation using tomotherapy (60 Gy/30 fractions), with concomitant chemotherapy consisting of temozolomide $\left(75 \mathrm{mg} / \mathrm{m}^{2} /\right.$ day $)$. After a 4 -week 


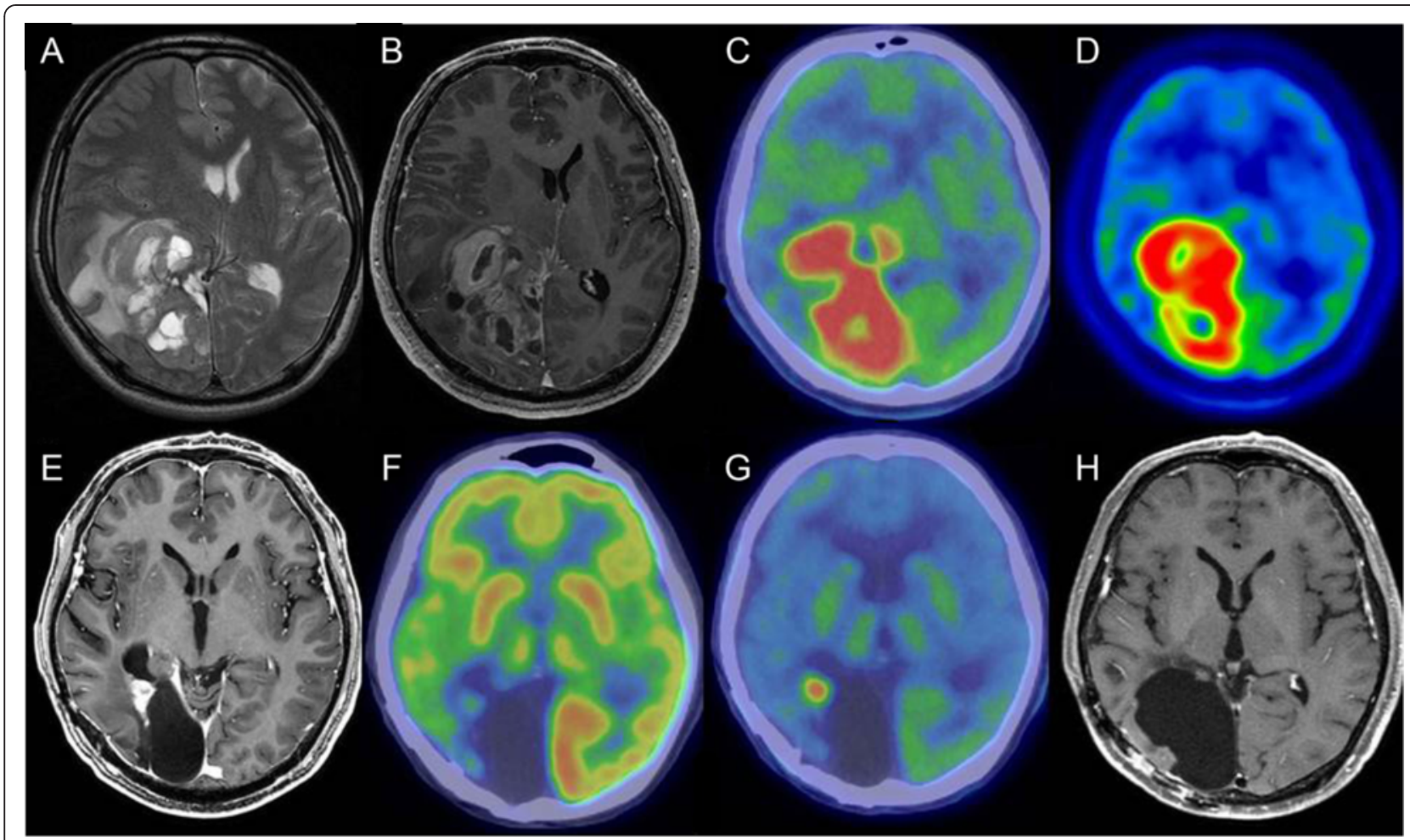

Figure 1 Magnetic resonance imaging (MRI) and positron emission tomography (PET). (A) T2-weighted image showing a huge multicystic mass in the right occipitoparietal area with marked surrounding edema and shift of the midline structures to the left side. (B) Each cyst wall and adjacent cortical mass was enhanced with contrast medium. (C) Fluorodeoxyglucose (FDG) PET showing high accumulation in the right occipitoparietal area. (D) Methionine (MET) PET showing high accumulation in the right occipitoparietal area. (E, F) MRI and PET findings at the time of recurrence. (E) Small enhanced mass adjacent to the cavity formed by removal of the tumor. (F) FDG-PET showing no accumulation in the mass. (G) MET-PET showing high accumulation in the mass. $(\mathbf{H})$ MRI 4 years after the first operation.

break, the patient received 19 cycles of adjuvant temozolomide $\left(150 \mathrm{mg} / \mathrm{m}^{2} /\right.$ day $)$ for 5 days every 28 days. A small contrast-enhancing lesion was seen on MRI close to an extraction cavity 22 months after the first operation. Because MET-PET showed a high accumulation in the mass, although none was detected using FDG-PET (Figure 1F, G), a second operation was performed, and the recurrence of GBM was diagnosed. Furthermore, the patient continues to receive 31 cycles of adjuvant temozolomide $\left(200 \mathrm{mg} / \mathrm{m}^{2} /\right.$ day) for 5 days every 28 days and is living without recurrence 4 years after the first operation (Figure 1H).

\section{Pathological findings}

Numerous atypical spindle cells were interspersed with gemistocytes (Figure 2A, D), and microvascular proliferation and pseudopalisading were present (Figure 2B, C). Tumor cells were highly positive for glial fibrillary acidic protein (GFAP; Figure 2E), and the Ki67 index was approximately $10 \%$ (Figure $2 \mathrm{~F}$ ). Expression of cytokeratins was undetectable in $\mathrm{EMA}^{+}$tumor cells (Figure 2G, H). Findings of tumor cells negative for epidermal growth factor receptor (EGFR) but positive for P53 are typical of secondary GBM (Figure 2I, J). Expression of the IDH1 $\mathrm{R} 132 \mathrm{H}$ mutant or the IDH1 R132H mutation was not detected using immunohistochemistry or NGS analysis, respectively (Figure $2 \mathrm{~K}$ ). In contrast, expression of the $B R A F$ V600E mutant was detected using immunohistochemistry, and the BRAF V600E mutation was detected using NGS (Figure 2L).

\section{DNA extraction and NGS}

DNA was extracted from formalin-fixed paraffinembedded (FFPE) sections using a NucleoSpin DNA FFPE XS Kit (Macherey-Nagel, Düren, Germany), and $225 \mathrm{ng}$ of each genomic DNA sample was subjected to target amplification and library preparation for NGS analysis using a HaloPlex Cancer Research Panel (ABL1, JAK2, AKT1, JAK3, ALK, KIT, AR KRAS, ATM, MAP2K1, BRAF, MAP2K4, CDKN2A, MET, CSF1R, NOTCH1, CTNNB1, NPM1, EGFR, NRAS, ERBB2, PDGFRA, ERBB4, PIK3CA, FANCA, PIK3R1, FANCC, PTEN, FANCF, RET, FANCG, RUNX1, FGFR1, SMAD4, FGFR2, SMO, FGFR3, SRC, FLT3, STK11, HRAS, TP53, IDH1, VHL, IDH2, WT1, MAP2K2; Agilent Technologies, Santa Clara, CA, USA) according to the manufacturer's 


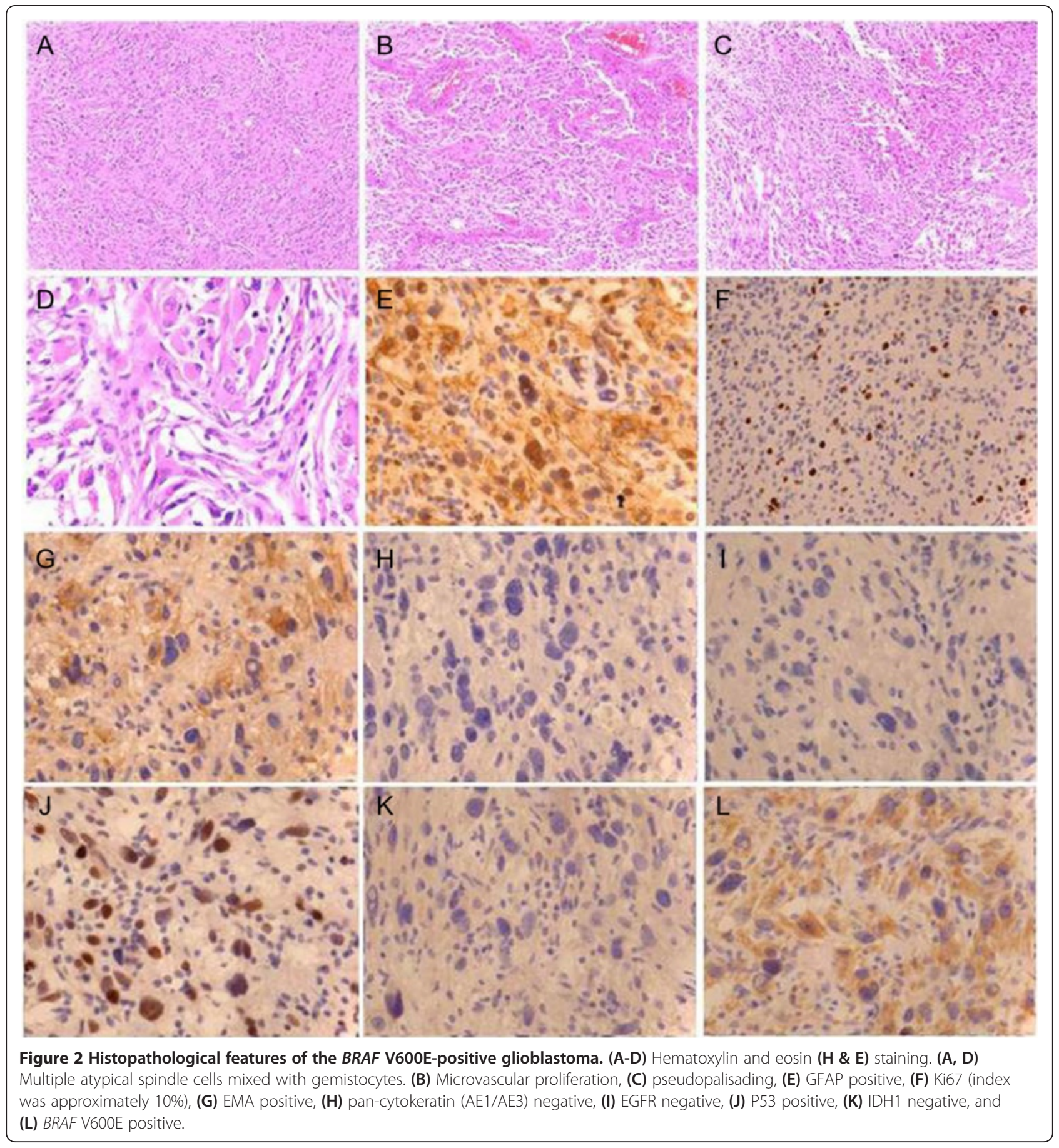

instructions. The target enrichment library pool was sequenced using a MiSeq (Illumina, San Diego, CA, USA). The sequence data were aligned, analyzed, and visualized using SureCall 2.0 software (Agilent Technologies).

\section{Immunohistochemistry}

Blocks of FFPE tumor sections were cut to a thickness of $4 \mu \mathrm{m}$, deparaffinized, and treated with an antigen unmasking solution (Immunosaver, Nissin EM Co. Ltd.,
Tokyo, Japan) at $90^{\circ} \mathrm{C}$ for $45 \mathrm{~min}$ and incubated with blocking solution $\left(3 \% \mathrm{H}_{2} \mathrm{O}_{2}\right)$ at room temperature. The anti-BRAF V600E (VE1) antibody (Ventana Medical Systems, Tucson, AZ, USA) was diluted 1:2,000 and incubated with sections for $16 \mathrm{~h}$ at $4^{\circ} \mathrm{C}$. Antibody-antigen reactions were detected using Bond Polymer Refine Detection reagents (Leica Biosystems, St. Louis, MO, USA). Additional sections were incubated for $1 \mathrm{~h}$ at room temperature in phosphate-buffered saline (PBS) 
with the antibodies as follows: ready-to-use formats of anti-GFAP clone GA5, anti-Ki67 clone SP6, anti-EMA clone E29, anti-pan-cytokeratin clone AE1/AE3, and anti-EGFR clone $31 \mathrm{G7}$ (all from Nichirei Bioscience, Tokyo, Japan); anti-P53 antibody diluted 1:100 (Dako, Glostrup, Denmark); or an anti-isocitrate dehydrogenase 1 (IDH1) R132H antibody clone H09 diluted 1:50 (Dianova, Hamburg, Germany). After incubation with primary antibodies, the sections were reacted with a peroxidase-conjugated secondary antibody (catalog number 424154, Nichirei Bioscience) for $1 \mathrm{~h}$ at room temperature and rinsed with PBS. The Histofine DAB kit (Nichirei Bioscience) was used to detect antigenantibody complexes.

\section{Conclusions}

The BRAF V600E mutation may occur at low frequency in adult c-GBM. This mutation was detected by the direct sequence method of Sanger method or immunohistochemistry. NGS techniques are in wide use because mutations are detected with greater sensitivity compared with Sanger sequencing $[7,8]$. Therefore, we have performed NGS analysis to detect BRAF mutations in tissues of 13 patients with c-GBM (11 primary and 2 secondary cases) who were treated at Hokuto Hospital. However, the BRAF V600E mutation was detected in only one case (7.7\%; Table 1$)$. The validity of the data is supported by the detection of BRAF V600E in the tumor tissue of the same patient after the tumor recurred.

When we reviewed the pathology of this case, the tumor was not an epithelioid or a giant cell GBM because foci with glandular and ribbon-like epithelial structures and multinucleated giant cells were not present. Classical GBM was confirmed by two expert neuropathologists (H.N. and S.T).

Although there is no detailed report of a $B R A F$ V600E-positive adult c-GBM, to our knowledge, there is a study of two such cases [9]. The tumors of both patients were located within the right parietal lobe. Interestingly, the tumor of our present patient was located in the right occipitoparietal lobe. Moreover, our patient with BRAF V600E-positive adult c-GBM was

Table 1 Result of next-generation sequencing analysis of glioblastoma

\begin{tabular}{|c|c|c|c|c|c|c|c|c|}
\hline Case & Age & Gender & Type & Gene & ID & Depth & Codon & Amino acid \\
\hline \multirow[t]{2}{*}{1} & 39 & $M$ & Primary & FANCA & rs2239359 & 264 & $\mathrm{Ggc} / \mathrm{Agc}$ & G501S \\
\hline & & & & STK11 & rs59912467 & 157 & $\mathrm{ttC} / \mathrm{ttG}$ & F354L \\
\hline \multirow[t]{3}{*}{2} & 49 & M & Primary & PDGFRA & & 458 & $\mathrm{cCg} / \mathrm{cGg}$ & p553R \\
\hline & & & & BRAF & rs121913227 & 311 & gTg/gAg & V600E \\
\hline & & & & FANCA & rs2239359 & 1,141 & $\mathrm{Ggc} / \mathrm{Agc}$ & G501S \\
\hline \multirow[t]{3}{*}{ Recurrence } & & & & PDGFRA & & 458 & $\mathrm{cCg} / \mathrm{cGg}$ & p553R \\
\hline & & & & BRAF & rs121913227 & 311 & gTg/gAg & V600E \\
\hline & & & & FANCA & rs2239359 & 1,141 & Ggc/Agc & G501S \\
\hline \multirow[t]{2}{*}{3} & 60 & M & Primary & $\mathrm{VHL}$ & rs369018004 & 8,156 & Ctg/Gtg & L129V \\
\hline & & & & FANCA & rs2239359 & 14,888 & Ggc/Agc & G501S \\
\hline 4 & 42 & $F$ & Secondary & FANCA & rs2239359 & 9,366 & $\mathrm{Ggc} / \mathrm{Agc}$ & G501S \\
\hline \multirow[t]{3}{*}{5} & 55 & M & Secondary & $\mathrm{IDH} 1$ & rs121913500 & 4,571 & $c G t / c A t$ & $\mathrm{R} 132 \mathrm{H}$ \\
\hline & & & & ATM & & 14,928 & Ggt/Agt & G2695S \\
\hline & & & & FANCA & rs2239359 & 18,284 & $\mathrm{Ggc} / \mathrm{Agc}$ & G501S \\
\hline 6 & 79 & M & Primary & FANCA & rs2239359 & 18,284 & $\mathrm{Ggc/Agc}$ & G501S \\
\hline \multirow[t]{2}{*}{7} & 80 & M & Primary & FANCA & rs2239359 & 3,313 & $\mathrm{Ggc} / \mathrm{Agc}$ & G501S \\
\hline & & & & STK11 & rs59912467 & 2,723 & $\mathrm{ttC} / \mathrm{ttG}$ & F354L \\
\hline 8 & 80 & M & Primary & FANCA & rs2239359 & 18,161 & $\mathrm{Ggc} / \mathrm{Agc}$ & G501S \\
\hline \multirow[t]{2}{*}{9} & 58 & M & Primary & FANCA & rs2239359 & 18,161 & Ggc/Agc & G501S \\
\hline & & & & STK11 & rs59912467 & 2,723 & $\mathrm{ttC} / \mathrm{ttG}$ & F354L \\
\hline 10 & 62 & M & Primary & FANCA & rs2239359 & 513 & $\mathrm{Ggc} / \mathrm{Agc}$ & G501S \\
\hline 11 & 66 & M & Primary & FANCA & rs2239359 & 8,387 & $\mathrm{Ggc} / \mathrm{Agc}$ & G501S \\
\hline \multirow[t]{2}{*}{12} & 63 & F & Primary & PDGFRA & rs2228230 & 2,959 & $\mathrm{gtC} / \mathrm{gtT}$ & V824 \\
\hline & & & & FANCA & rs2239359 & 7,658 & $\mathrm{Ggc} / \mathrm{Agc}$ & G501S \\
\hline 13 & 62 & M & Primary & FANCA & rs2239359 & 8,036 & $\mathrm{Ggc} / \mathrm{Agc}$ & G501S \\
\hline
\end{tabular}


alive when this manuscript was submitted, 4 years after the first operation. In contrast, the patients studied by Chi et al. [9] survived for 19 and 36 months. The IDH1 mutation serves as a good prognostic factor for patients with GBM but was not detectable using NGS or immunohistochemical analyses. These data are consistent with the results of a study of two patients with BRAF V600Epositive c-GBM [9].

Although the number of patients was small, these three patients with BRAF V600E-positive GBM survived relatively longer compared with patients without this mutation. Therefore, tumors with the BRAF V600E mutation may represent a more favorable subtype of GBM. More patients with GBM must be analyzed to conclude that the BRAF V600E mutation is a potential prognostic marker for GBM. Moreover, inhibitors of B-Raf protein kinase activity may serve as efficacious drugs for treating patients with BRAF V600E-positive GBM $[10,11]$. Therefore, we suggest that we should perform routine genetic testing of BRAF V600E mutation, which might provide effective alternatives to treat patients with GBM.

\section{Consent}

Written informed consent was obtained from the patient for publication of this case report and any accompanying images. A copy of the written consent is available for review by the Editor-in-Chief of this journal.

\section{Competing interests}

The authors declare that they have no competing interests.

\section{Authors' contributions}

YT, HI, and AT participated in the design of this study. TS performed NGS, and TA, HN, and ST performed the histological examination. SY, KN, WI, IH, and $\mathrm{HK}$ collected important background information and helped to draft the manuscript. All authors read and approved the final manuscript.

\section{Acknowledgements}

We thank the patient for consenting publication of this case report.

\section{Author details}

'Department of Neurosurgery, Hokuto Hospital, 7-5, Inada, Obihiro, Hokkaido 080-0039, Japan. ${ }^{2}$ Department of Pathology, Hokuto Hospital, 7-5, Inada, Obihiro, Hokkaido 080-0039, Japan. ${ }^{3}$ Department of Biology and Genetics, Laboratory of Cancer Medical Science, Hokuto Hospital, 7-5, Inada, Obihiro, Hokkaido 080-0039, Japan. ${ }^{4}$ Department of Translational Pathology, Hokkaido University Graduate School of Medicine, N15, W7, Kita-ku, Sapporo, Hokkaido 060-8638, Japan.

Received: 7 January 2015 Accepted: 23 February 2015

Published online: 11 March 2015

\section{References}

1. Schlaepfer DD, Jones KC, Hunter T. Multiple Grb2-mediated integrin-stimulated signaling pathways to ERK2/mitogen-activated protein kinase: summation of both c-Src- and focal adhesion kinase-initiated tyrosine phosphorylation events. Mol Cell Biol. 1998;18:2571-85.

2. Dias-Santagata D, Lam Q, Vernovsky K, Vena N, Lennerz JK, Borger DR, et al. BRAF V600E mutations are common in pleomorphic xanthoastrocytoma: diagnostic and therapeutic. PLoS One. 2011;6:e17948.

3. Dougherty MJ, Santi M, Brose MS, Ma C, Resnick AC, Sievert AJ, et al. Activating mutations in BRAF characterize a spectrum of pediatric low-grade gliomas. Neuro Oncol. 2010;12:621-30.
4. Koelsche C, Wöhrer A, Jeibmann A, Schittenhelm J, Schindler G, Preusser M, et al. Mutant BRAF V600E protein in ganglioglioma is predominantly expressed by neuronal tumor cells. Acta Neuropathol. 2013;125:891-900.

5. Schindler G, Capper D, Meyer J, Janzarik W, Omran H, Herold-Mende C, et al. Analysis of BRAF V600E mutation in 1320 nervous system tumors reveals high mutation frequencies in pleomorphic xanthoastrocytoma, ganglioglioma and extra-cerebellar pilocytic astrocytoma. Acta Neuropathol. 2011;121:397-405.

6. Kleinschmidt-DeMasters BK, Aisner DL, Birks DK, Foreman NK. Epithelioid GBMs show a high percentage of BRAF V600E mutation. Am J Surg Pathol. 2013;37:685-98.

7. Neveling K, Feenstra I, Gilissen C, Hoefsloot LH, Kamsteeg EJ, Mensenkamp $A R$, et al. A post-hoc comparison of the utility of sanger sequencing and exome sequencing for the diagnosis of heterogeneous diseases. Hum Mutat. 2013;34:1721-6.

8. Tuononen K, Mäki-Nevala S, Sarhadi VK, Wirtanen A, Rönty M, Salmenkivi K, et al. Comparison of targeted next-generation sequencing (NGS) and real-time PCR in the detection of EGFR, KRAS, and BRAF mutations on formalin-fixed, paraffin-embedded tumor material of non-small cell lung carcinoma-superiority of NGS. Genes Chromosomes Cancer. 2013;52:503-11.

9. Chi AS, Batchelor TT, Yang D, Dias-Santagata D, Borger DR, Ellisen LW, et al. BRAF V600E mutation identifies a subset of low-grade diffusely infiltrating gliomas in adults. J Clin Oncol. 2013;10:233-6.

10. Robinson GW, Ori BA, Gajjar A. Complete clinical regression of a BRAF V600E-mutant pediatric glioblastoma multiforme after BRAF inhibitor therapy. BMC Cancer. 2014;14:258.

11. Nicolaides T, Li H, Solomon D, Hariono S, Hashizume R, Barkovich $\mathrm{K}$, et al. Targeted therapy for BRAFV600E malignant astrocytoma. Clin Cancer Res. 2011;17:7595-604.

\section{Submit your next manuscript to BioMed Central and take full advantage of:}

- Convenient online submission

- Thorough peer review

- No space constraints or color figure charges

- Immediate publication on acceptance

- Inclusion in PubMed, CAS, Scopus and Google Scholar

- Research which is freely available for redistribution 